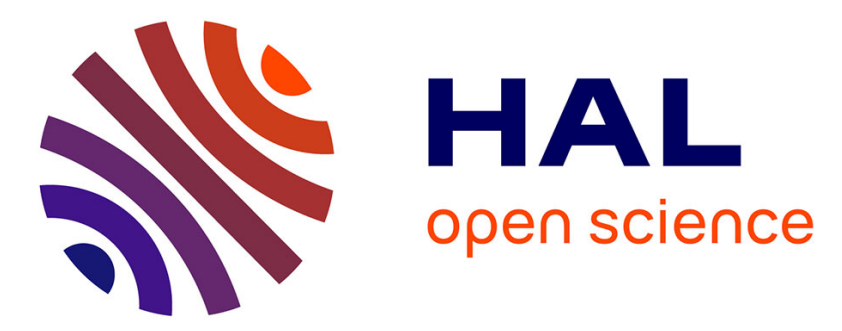

\title{
Temporal Bag-of-Words - A Generative Model for Visual Place Recognition using Temporal Integration
}

Hervé Guillaume, Mathieu Dubois, Frenoux Emmanuelle, Philippe Tarroux

\section{To cite this version:}

Hervé Guillaume, Mathieu Dubois, Frenoux Emmanuelle, Philippe Tarroux. Temporal Bag-of-Words A Generative Model for Visual Place Recognition using Temporal Integration. VISAPP - International Conference on Computer Vision Theory and Applications - 2011, INSTICC, Mar 2011, Vilamoura, Portugal. hal-00765798

\section{HAL Id: hal-00765798 https://hal.inria.fr/hal-00765798}

Submitted on 16 Dec 2012

HAL is a multi-disciplinary open access archive for the deposit and dissemination of scientific research documents, whether they are published or not. The documents may come from teaching and research institutions in France or abroad, or from public or private research centers.
L'archive ouverte pluridisciplinaire HAL, est destinée au dépôt et à la diffusion de documents scientifiques de niveau recherche, publiés ou non, émanant des établissements d'enseignement et de recherche français ou étrangers, des laboratoires publics ou privés. 


\title{
TEMPORAL BAG-OF-WORDS: A GENERATIVE MODEL FOR VISUAL PLACE RECOGNITION USING TEMPORAL INTEGRATION
}

\author{
Hervé Guillaume $^{* \dagger}$, Mathieu Dubois* ${ }^{* \dagger}$, Emmanuelle Frenoux ${ }^{\dagger *}$ and Philippe Tarroux ${ }^{\dagger \ddagger}$ \\ * Univ Paris-Sud, Orsay, F-91405 \\ ${ }^{\dagger}$ LIMSI - CNRS, B.P. 133, Orsay, F-91403 \\ $\ddagger$ Ecole Normale Supérieure, 45 rue d'Ulm, Paris, F-75230 \\ \{herve.guillaume, mathieu.dubois, emmanuelle.frenoux, philippe.tarroux\}@limsi.fr
}

Keywords: Place recognition, Bag-of-Words, Temporal integration, Generative learning.

\begin{abstract}
This paper presents an original approach for visual place recognition and categorization. The simple idea behind our model is that, for a mobile robot, use of the previous frames, and not only the one, can ease recognition. We present an algorithm for integrating the answers from different images.

In this perspective, scenes are encoded thanks to a global signature (the context of a scene) and then classified in an unsupervised way with a Self-Organizing Map. The prototypes form a visual dictionary which can roughly describe the environment. A place can then be learnt and represented through the frequency of the prototypes. This approach is a variant of Bag-of-Words approaches used in the domain of scene classification with the major difference that the different "words" are not taken from the same image but from temporally ordered images. Temporal integration allows us to use Bag-of-Words together with a global characterization of scenes. We evaluate our system with the COLD database. We perform a place recognition task and a place categorization task. Despite its simplicity, thanks to temporal integration of visual cues, our system achieves state-of-the-art performances.
\end{abstract}

\section{INTRODUCTION}

Mobile robot localization has been an intensive field of study in the past decades (see (Filliat and Meyer, 2003)). Traditional approaches have focused on metric localization (i.e. the ability to position objects in a common coordinate frame) or topological localization (i.e. the ability to build a graph of interesting places). However in those approaches, a place does not necessary coincide with the human concept of rooms. In this paper the word "place" designates a particular room such as office 205, the corridor of the second floor, etc. Recently research had focused on semantic place recognition which is the ability for a mobile robot to recognize the room (kitchen office) it is currently in and the nature of its environment. The knowledge of the semantic context allows to draw hypothesis on the identity of perceived objects (see (Torralba, 2003)). There is a huge semantic gap between the human notion of room and sensor measurements available to the robot. Therefore, contrary to SLAM or topological mapping, semantic place recognition is a supervised problem.

Several solutions have been proposed for place recognition and place categorization using vision alone or combined with several types of telemeters (laser, sonar) (see (Pronobis et al., 2010)). Vision provides richer information than telemeters which is an essential advantage for fine discrimination and categorization of places according to semantic criteria. That's why vision receives an increasing attention in the community of place recognition.

Nevertheless, visual recognition and moreover visual categorization of places is still an open problem due to at least three major problems. First, the size of the input space is large due to the size of images. Second, the inter-class distance is small (different classes share common visual features) while the intra-class distance is large (for instance, an office in a laboratory can look very different from an office in another laboratory). Last but not least, applications on a real robot enforce on-line and real-time algorithms (potentially with low computational resources).

Most works tackle the problem of visual place 
recognition as a problem of scene classification i.e. each frame taken by the robot is assigned to a place. We think that place recognition is a slightly different problem as a single frame may be intrinsically ambiguous, non-informative or even misleading for place recognition. Imagine that the robot is in an office but faces a window. The scene will contain trees, cars, other buildings, etc. A scene classification algorithm might assign the label "outside" to the image. This example illustrates very well the difference between scene classification (which basically answers the question "What am I seeing right now?") and place recognition (which answers the question "According to what I am seeing, in which place am I?").

This article presents two contributions. First, we develop a method which takes advantage the exploratory capacity of a robot. The main idea is to collect several cues during the exploration to disambiguate the current scene. While a scene can be ambiguous a collection of scenes is generally not: for instance in the case of a robot facing a window at time $t$, the robot might at time $t+1$ face a stair indicating that it is in the entrance of the laboratory.

Second, we present a system for place recognition which illustrates this idea. Our system can be briefly described as follows. Each scene is characterized thanks to a coarse global vector (the visual context) and each vector is quantized with a vector quantization algorithm. Thus, we obtain a visual dictionary (or codebook) which allow us to describe the environment in terms of visual words (i.e. visual scene prototypes). During the learning stage, each place is described by the respective frequencies of the prototypes. Finally, during the recognition phase, the classification output is obtained thanks to the last $L$ observations done during exploration. Our method can be seen as a modification of the Bag-of-Words algorithm (see (Csurka et al., 2004)) with two major differences: 1. The different words are not taken from the same image but from temporally ordered images 2 . Temporal integration allows us to use Bag-of-Words together with a global characterization of scenes.

The rest of the paper is organized as follows. Section 2 presents related work. Section 3 presents the descriptor used and the constitution of the visual dictionary. Section 4 presents the place models and the Bayesian formalism used for cue integration. Section 5 shows the results of our system in a place recognition task and section 6 in a place categorization task.

\section{RELATED WORKS}

The vast majority of works on place recognition use techniques developed for scene classification. Some of them rely on prior object identification (see for instance (Vasudevan and Siegwart, 2008)). However most of the recent scene classification and place recognition systems bypass this step due to its high complexity and because high performances can be reached without it.

In this case, the authors usually compute various descriptors on laser range scans (see for instance (Mozos et al., 2005; Mozos, 2008)) or on images (using either perspective or omnidirectional cameras). Multi-modal approaches also exist (see for instance (Pronobis et al., 2010)). Among visionbased methods we can distinguish between methods using global images features (see (Walker and Malik, 2004:Oliva and Torralba, 2001; Pronobis et al., 2006. Orabona et al., 2007; Torralba et al., 2003)) and methods using local descriptors (Ullah et al., 2008, Filliat, 2008; Ni et al., 2009) usually computed around interest points. Colours and orientations are the most used features.

One such local method is the Bag-of-Words model (BoW) (see (Gokalp and Aksoy, 2007; Fei-Fei and Perona, 2005, Lazebnik et al., 2006)). The main idea is to define a visual dictionary by mean of a vector quantization algorithm. The major advantage is that quantization decreases the computational cost of learning while keeping a good classification rate. BoW has been used in the domain of semantic place recognition (see (Filliat, 2008) for instance).

In (Pronobis and Caputo, 2007) the authors use a confidence criterion to compute several cues from the same image. The idea is first to compute a simple cue to classify the image. If the confidence is not high enough then compute another cue and combine those decisions. This process is repeated until confidence is sufficiently high (or no more cues are available). Again, contrary to the work presented here, recognition is carried out using only one image.

In (Pronobis et al., 2010), the authors use a simple spatio-temporal accumulation process to filter the decision of a discriminative confidence-based place recognition system (which uses only one image to recognize the place). The responses of the system are accumulated spatially and temporally along the robot's trajectory, thanks to odometric information, creating a sparse 3D-histogram. The answer of the system is then the average over space of the accumulated responses. The size of the bins must be adjusted so that each bin roughly corresponds to a single viewpoint. One problem with this method is that 
the system needs to wait some time before giving a response. Also special care must be taken to detect places boundaries.

Some authors (Mozos, 2008: Torralba et al., 2003) use the topology of the environment to predict the transition between two places and increase general performance. These works use a Hidden Markov Model (HMM) where each place is a hidden state of the HMM and the feature vector stands for the observations. One drawback, as there is no quantization, is that the input space is continuous and highdimensional. The learning procedure is then computationally expensive.

The work that is the closest to our is (Wu et al., 2009). The author proposed a system based on quantized descriptors (i.e. a BoW model) in conjunction with Bayesian Filtering. Our method can be seen as a simplification of this method since we don't need to learn or make assumptions on the probabilities of the transition between places.

Compared to the previous works, one originality of our system is to combine BoW modelling and global features instead of local ones. This is made possible because visual cues are collected over time instead of being collected over the current visual scene.

\section{OVERVIEW OF THE SYSTEM}

Our system is an adaptation of the classic BoW system. Each image is described by a global signature (i.e. a numerical feature vector): at each time step $t$, the input image $I(t)$ is presented to the system and its signature is computed. There are two learning phases.

The first step is to train a vector quantization algorithm. Once this is done, an image can be mapped to a predetermined vector (i.e. a prototype). One image is then represented by an integer $o(t)=k \in\{1 . . S\}$ which identifies the prototype ( $S$ is the number of prototypes). The set of prototypes is the vocabulary that will be used for learning places.

Then learning of the place model can take place. Training is supervised so each observation is labelled with the name of the place $c_{i} \in C$ we are currently in $(C$ denotes the set of places in the current environment).

\subsection{Image signatures}

To characterize the images we use two recent global descriptors that have been developed in the context of place recognition: GIST (see (Oliva and Torralba, 2001)) and CENTRIST (see (Wu et al., 2009)). Those descriptors are global i.e. they use all the pixels to compute the signature (there is no extraction of interest points). Thus we say that they capture the visual context of the image.

GIST has been proposed in Oliva and Torralba, 2001) as a global holistic representation of a scene. It was successfully used for outdoor scene classification. GIST is based on the output of a Gabor-like filter bank (we use 4 scales and 6 orientations) applied on each colour channel. The $4 \times 6 \times 3=72$ resulting images are evenly divided into $4 \times 4$ sub-windows. The output of the filter is averaged on each subwindow and the resulting vectors are concatenated. We used the $\mathrm{C}$ implementation proposed in (Douze et al., 2009). To further reduce the dimensionality we project the $4 \times 4 \times 72=1152$-dimensional vector onto the 80 first principal components (which explain more than $99 \%$ of the variance) computed on a database made of $1 / 20$ images in the COLD database. The signature captures the most significant spatial structure in the image.

CENsus TRansform hISTogram (CENTRIST) was proposed by (Wu et al., 2009) for a place categorization task. First, the edges of the image are computed using a first-order Sobel filter. The image is then transformed using Census Transform (CT). This transformation is similar to $L B P_{8,1}$ (see (Ojala et al., 2002)) and captures the local intensity pattern of the edges. This transformation is robust to illumination and gamma changes. The CENTRIST descriptor is the 256-bins histogram of the CT values. Note that contrary to (Wu et al., 2009) we do not divide the image in sub-windows. Instead we use only one histogram for all the pixels in the image.

\subsection{The Self-Organizing Map and its training}

The vector quantization algorithm chosen in this paper is the Self-Organizing Map (SOM) (Kohonen, 1990). It consists in a neural network where neurons are disposed on a lattice. In this article we will investigate square maps of different sizes $S$ (ranging from $5 \times 5$ to $20 \times 20$ ) with a toroidal topology and a Gaussian neighbourhood. Each neuron (or unit) holds a weight vector (of the same size than the signature).

The SOM needs to be trained with the input vectors (this step should not be confused with the supervised learning of the places' models). At the end of the learning phase the SOM describes a discrete approximation of the distribution of training samples. This process has been shown to form clusters of similar images (see (Guillaume et al., 2005)). In the following of this article we shall call "visual prototypes" 
or "syntactic categories" the SOM's neurons. The set of all the prototypes form a dictionary of visual scenes.

In the current set-up the training of the SOM is performed off-line. The off-line training is supposed to give innate syntactic categories. That's why we have trained the SOMs with a representative sample of the visual environment made of $1 / 3$ the images of the COLD database (see section 5 .

\subsection{Place models}

Once the training of the SOM done we can start to learn the place models. Because the vocabulary is discrete and finite we can use a non-parametric approach. We have chosen to use Naive Bayes Classifier (NBC). Despite being a very simple generative model the NBC is able to compete with most discriminative algorithms (see (Ng and Jordan, 2002)).

We learn one classifier per place. The model of a place is simply the distribution of the visual prototypes found during learning which approximates the likelihood $P\left(o(t) \mid c_{i}\right)$. To avoid null values due to small training set we use the Laplace estimator.

Another interesting feature of our algorithm is that it is incremental and on-line (once the training of the SOM is done) and has a low computational complexity due to the discretization of the input space. The advantage over the work presented in (Mozos, 2008, Torralba et al., 2003) is that there are no hidden states. In our case the mapping of an image to a prototype gives its syntactic category.

\section{FRAMEWORK FOR CUE INTEGRATION}

We present here the framework for the temporal integration of cues which is the main contribution of this paper. This framework is also based on the Bayes formula but this should not be confused with the NBC learning and training. As discussed in previous sections it is interesting for the robot to combine the informations from different images to take advantage of the spatial extension of a place and its ability to explore it.

During learning, each image is processed separately. During recognition the robot will gather a set of observations with a sliding window of size $L$ :

$$
\mathbf{O}^{L}(t)=\{o(t-L+1), \ldots, o(t-1), o(t)\}
$$

We seek for the place $c^{*}$ which maximizes the $a$ posteriori probability for a given sequence $\mathbf{O}^{L}(t)$ :

$$
c^{*}=\underset{c_{i} \in C}{\operatorname{argmax}} P\left(c_{i} \mid \mathbf{O}^{L}(t)\right)
$$

If we consider that the $L$ observations are independent and using Bayes' rule we have:

$$
\begin{aligned}
c^{*} & =\underset{c_{i} \in C}{\operatorname{argmax}} \prod_{t^{\prime}=t-L+1}^{t} P\left(c_{i} \mid o\left(t^{\prime}\right)\right) \\
& =\underset{c_{i} \in C}{\operatorname{argmax}} \prod_{t^{\prime}=t-L+1}^{t} P\left(o\left(t^{\prime}\right) \mid c_{i}\right) P\left(c_{i}\right)
\end{aligned}
$$

Considering that the different places are equiprobable we can apply the maximum likelihood rule:

$$
c^{*}=\underset{c_{i} \in C}{\operatorname{argmax}} \prod_{t^{\prime}=t-L+1}^{t} P\left(o\left(t^{\prime}\right) \mid c_{i}\right)
$$

The temporal integration consists in multiplying the likelihood of each place (given by the NBC) over a window made of the last $L$ observations and then searching for the maximum. The likelihood of the observation at time-step $t^{\prime}$ for a place $c_{i}, P\left(o\left(t^{\prime}\right) \mid c_{i}\right)$, is given by the NBC trained in the previous section but our method can be applied to any probabilistic classifier. To avoid numerical underflows we use the loglikelihood.

In Equation 4 the window is made of the last $L$ observations. During this observation period the robot can move from one place to another and therefore the temporal integration window can contain cues from different places (this is similar to the threshold-detection problem mentioned in (Pronobis et al., 2010)). To avoid this, we use an explicit "reset" mechanism during recognition: an oracle informs the system when it changes from one place to another; the system then restarts the recognition with an empty window. The size of the window is then dynamic and $L$ is just an upper bound.

A special case is when the size of the windows is infinite. This means that there is no constraint on the size of the integration window except the explicit reset when the robot moves from one place to another.

Note that the computational cost of integration is very low and that it doesn't need additional learning. Moreover the system is any-time: we are still able to give a response at each time-step (we don't have to wait $L$ time-steps to give an answer) although the correctness should increase as more observations are integrated.

The length of the integration window $L$ is an important parameter of our system. In the following sections we will evaluate the performance of our system and the influence of $L$ on it. 


\section{RECOGNITION OF INSTANCES}

\subsection{Experimental design}

Experiments were carried out on the COLD (COsy Localization Database) database (see (Pronobis and Caputo, 2009)). This database was designed for evaluating vision-based place recognition systems for mobile platforms in realistic settings and to test the robustness against different kinds of variations. The sequences where acquired in different laboratories across Europe (Saarbruecken, Freibug and Ljubljana). Some laboratories were divided in two parts (denoted as "A" and "B"). In each laboratory two paths were explored (denoted "standard" and "extended"). Each path was acquired under different illumination conditions (night, cloudy and sunny) and several times. The sampling frequency was $5 \mathrm{~Hz}$. All experiments were carried out with the perspective images.

For fair comparison we performed the same experiments as in (Ullah et al., 2008). The task during these experiments was to recognize a room, seen during training, when imaged under different conditions, i.e. at a different time and/or under different illumination settings. For each experiment, training set consisted of one sequence taken in one laboratory, and testing was done on sequences acquired in the same laboratory, under various conditions. With these experiments it was possible to verify robustness to dynamic changes as well as to geographic changes, as the parameters of the algorithms were always the same. The results were averaged for all permutations of the training and testing sets (ensuring that training and testing were always performed on different sequences). Sequences where some places are missing where ignored 1 .

\subsection{Overall performances}

Figure 1 shows the best results obtained for the GIST and CENTRIST descriptors. Those results were obtained with the SOM size fixed to $S=20 \times 20$ and using an infinite integration window.

The overall results are good. For instance, the average recognition rate when trained and tested under the same illumination condition (congruent condition) can be seen in table1. Those results are better than the one described in (Ullah et al., 2008).

Both descriptors show a good robustness to varying illumination conditions with a slight advantage to CENTRIST. This can be seen in figure 1; the difference between classification rates in congruent case, in

\footnotetext{
${ }^{1}$ See http://cogvis.nada.kth.se/COLD/bugs.php for the list of erroneous sequences
}

Table 1: Average recognition rate in congruent conditions for GIST and CENTRIST descriptors compared to (Ullah et al., 2008). For each laboratory, the best result is in bold. The results show that GIST performs slightly better than CENTRIST especially in Ljubljana.

\begin{tabular}{|l|c|c|c|}
\hline & GIST & CENTRIST & Ullah et al., 2008) \\
\hline Saar. - Std & $\mathbf{9 3 . 7 \%}$ & $91.1 \%$ & $90.5 \%$ \\
\hline Saar. - Ext & $84.5 \%$ & $\mathbf{8 4 . 9 \%}$ & $83.8 \%$ \\
\hline Frei.- Std & $91.7 \%$ & $\mathbf{9 1 . 9 \%}$ & $85.6 \%$ \\
\hline Frei.- Ext & $\mathbf{8 9 . 6 \%}$ & $86.2 \%$ & $81.8 \%$ \\
\hline Ljub. - Std & $\mathbf{9 0 . 7 \%}$ & $81.0 \%$ & $90.4 \%$ \\
\hline Ljub. - Ext & $\mathbf{8 7 . 7 \%}$ & $77.6 \%$ & $85.5 \%$ \\
\hline
\end{tabular}

one hand, and non-congruent cases, in the other hand, is smaller for CENTRIST (figure 1(b)) than for GIST (figure 1(a)).

If we take into consideration all the cases, our system performs slightly better for extended sequences but slightly less well than the one described in (Ullah et al., 2008) for standard sequences. A notable exception is the Ljubljana laboratory where we perform less well especially the GIST descriptor on "night" sequences. A possible explanation is that night sequences in the Ljubljana laboratory are very dark. A global signature is more sensitive to the global illumination level than a local one (such as GIST used in (Ullah et al., 2008)). For instance the GIST signature (and the prototypes) for those sequences will be different from the ones for "sunny" or "cloudy" sequences which explains the low recognition rate. As said previously CENTRIST is more robust to illumination changes thanks to the $\mathrm{CT}$.

\subsection{Influence of the size of the integration window}

The main point of this paper is to study the influence of the size of the integration window $L$ and possibly to show a positive impact on performance. To do so we repeated the experiment above with several values of $L$ and computed the average recognition rate. To avoid interaction between the robustness to illumination and the effect of $L$ we study the case of congruent illumination. Figure 2 shows the influence of the length of the integration window $L$ on the performance for each type of signature.

With only one image the system is a pure scene classification algorithm. The performances are rather poor $(58 \%$ of correct recognition for CENTRIST, $71 \%$ for GIST) which suggests that the visual prototypes given by the SOM are not sufficient for place recognition. The average classification rate increases quickly as $L$ goes to $+\infty$. For example with GIST the average performance rises from $71 \%$ to nearly 

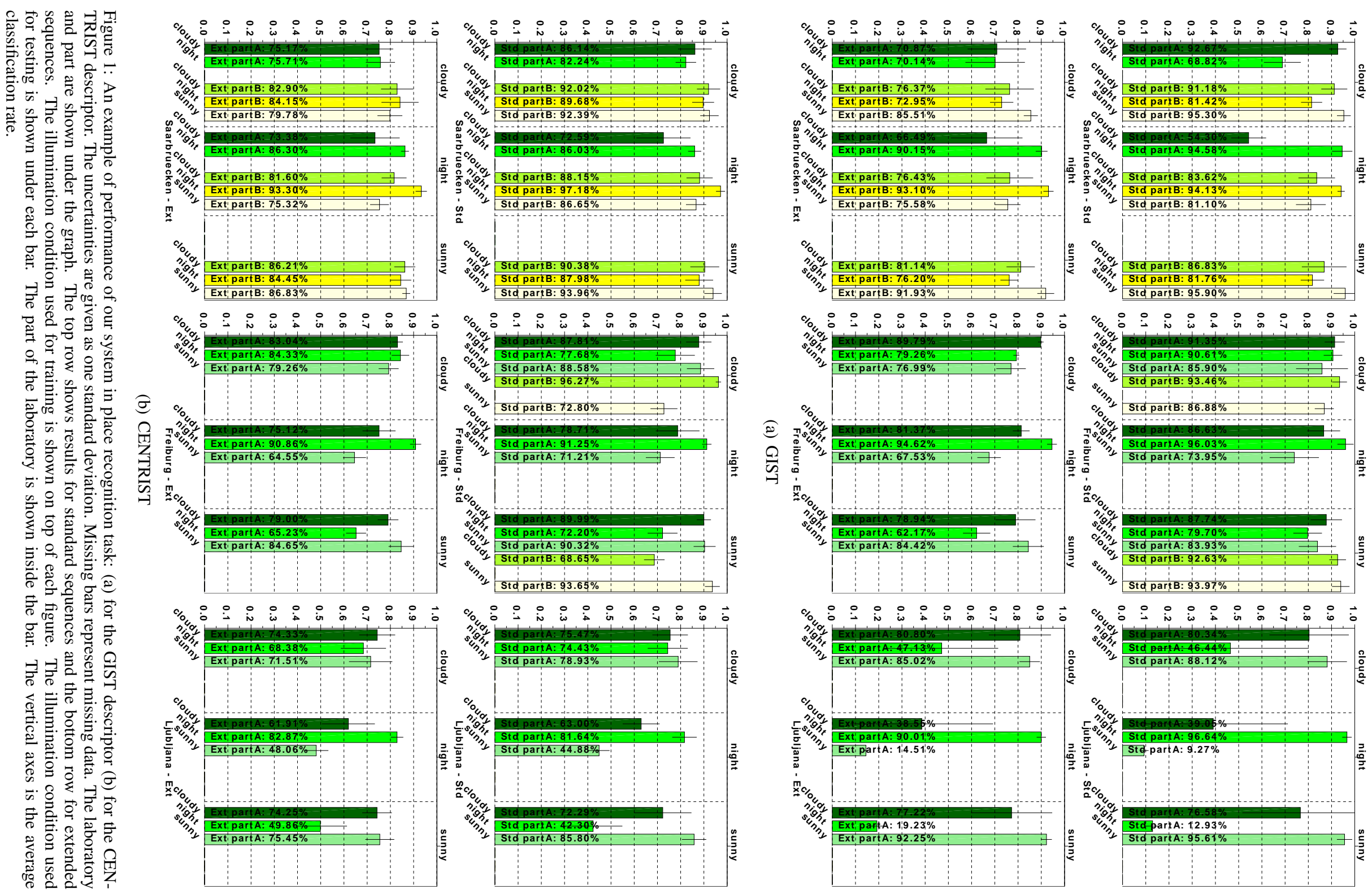

ڤ
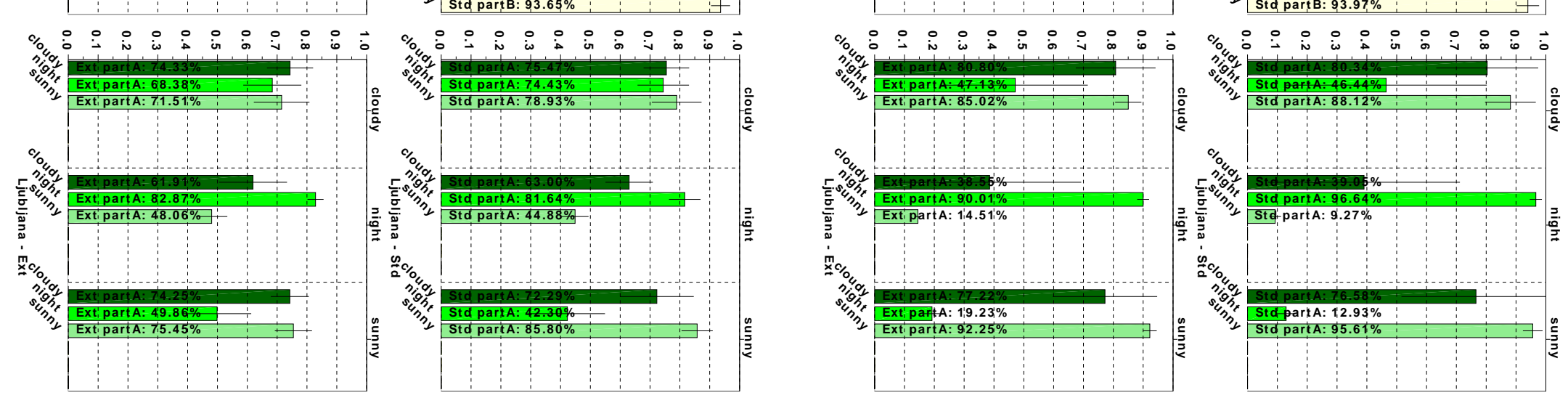


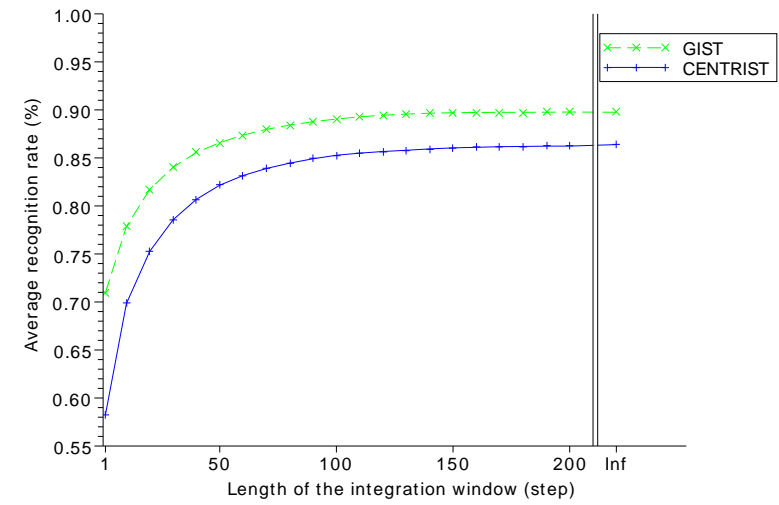

Figure 2: Influence of the size of the integration window on the average classification rate for each signature.

$90 \%$ (increase of 19\%). With CENTRIST the average recognition rises from $58 \%$ to $84 \%$ (increase of $26 \%$ ). With a window of length 50 the average recognition rate is already higher than $80 \%$ for CENTRIST and $85 \%$ for GIST.

Given the simplicity of our system which uses only a global and very coarse description of each scene this demonstrates the benefits of temporal integration for place recognition.

\section{CATEGORIZATION OF PLACES}

In this experiment we test the system on a place categorization task. Place categorization is a much harder task because the goal is to assign the label "office" to any office in the test set while the system has been trained on other offices. Therefore it is interesting to assess the performance of our system on such a task and to study the influence of the parameters.

Again, for fair comparison, we reproduce the experimental design of (Ullah et al., 2008). Here the algorithm is trained to recognize four different room categories (corridor, printer area, two-persons office and bathroom), all available in the standard sequences of part " $A$ " of each laboratory. The algorithm is trained on two sequences taken from two laboratories. Testing was performed on sequences taken at the third remaining laboratory. In this case the training and testing illumination condition are the same.

Figure 3 shows results obtained for the 2 signatures with a SOM size of $S=5 \times 5$ for GIST and $15 \times 15$ for CENTRIST. In both cases we used an infinite integration window.
Like in (Ullah et al., 2008) the corridor is always the best recognized category but CENTRIST also performed well on the two-persons office. Averaged across all the tests CENTRIST achieve classification rates of $84.72 \%$ for the corridor, $47.96 \%$ for the two-persons office, $37.16 \%$ for the printer area and $28.18 \%$ for the bathroom. The performances of GIST are $72.21 \%, 30.95 \%, 22.12 \%$ and $17.67 \%$ respectively.

Those results are better than those reported in (U1lah et al., 2008) (76.73\% for the corridor, far lower for other places). (Wu et al., 2009) obtained interesting results but on a different dataset.

To evaluate the influence of the two parameters $S$ and $L$ we performed the same experiment for $S$ ranging from $5 \times 5$ to $20 \times 20$ and $L$ ranging from 1 to $+\infty$. To assess the system we use the average recognition rate.

Figure 4 shows the results for the 2 descriptors. GIST didn't perform well on this categorization task: the responses are barely above the level of chance (25\% since we have 4 categories). This result is coherent with the low performances of GIST in indoor scene categorization task (see (Quattoni and Torralba, 2009)). As CENTRIST was designed for place categorization we expected good results for this descriptor: the average recognition rate reaches $50 \%$ for $S=15$ and infinite integration window which is higher than the results reported in (Ullah et al., 2008).

Interestingly, unlike the case of recognition, the effect of the size of the SOM is non-monotonic. This may be explained by the fact that on one hand a smaller SOM implies less visual prototypes and then may ease categorization but on the other hand a smaller SOM decreases the ability to discriminate between different rooms which may lead to confusion between different categories.

The effect of $L$ on this task is clear for CENTRIST: a larger window always give better results which shows the interest of our integration method.

However a closer look at data shows that there is a high false-positive rate. In fact the high classification rate can be explained because one or two classifiers win most of the time (the classifiers for the toilets and the corridor in our case). This also explains the large deviation that we observe.

\section{CONCLUSION}

The main contribution of this paper is to show the advantage of temporal integration of visual cues for place recognition. This idea is deeply linked with the spatial nature of a place and the abilities of a robot 


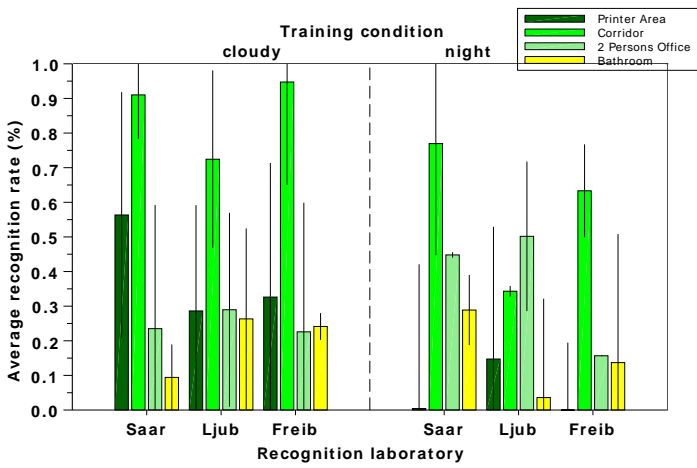

(a) GIST

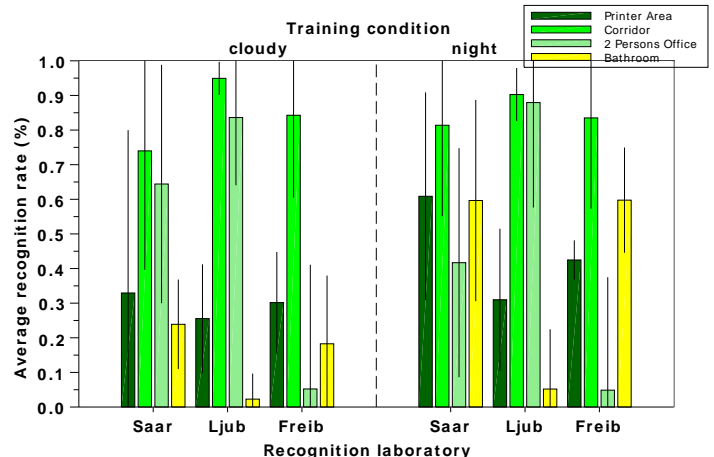

(b) CENTRIST

Figure 3: Example of results obtained (a) with GIST and a SOM of size $S=5 \times 5$ (b) with CENTRIST and a SOM of $S=15 \times 15$. In both cases we used an infinite integration window. The uncertainties are given as one standard deviation. The illumination condition used for training and testing is shown on top of the figure. The laboratory used for training is shown under.

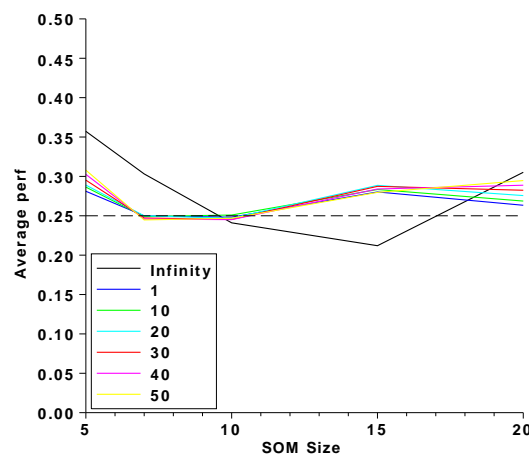

(a) GIST

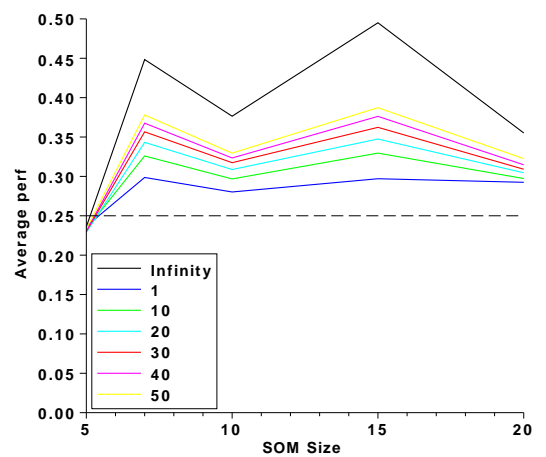

(b) CENTRIST

Figure 4: Influence of the size of the SOM and the integration window for signatures computed on the categorization task: (a) for the GIST descriptor (b) for the CENTRIST descriptor. The line of chance is indicated by the dashed line.

to explore it. It allows to describe the visual environment in terms of global content while keeping good recognition rate and can be used for different systems.

We have presented a BoW-like system that takes advantage of this approach in several ways. Visual environment can be described thanks to coarse descriptors and a small number of visual prototypes based on global features (i.e. a dictionary of visual scenes). Combined with cue integration they form a generative, any-time, incremental and on-line place recognition system. The results on the place recognition task have shown that the system compares well with state-of-the-art systems. However, despite good performance in some cases, place categorization is still an open problem.

From a computational point-of-view the most de- manding phase is the computation of the visual descriptor. CENTRIST is relatively easy to compute. GIST is more challenging but fast implementation exists. As the other components of the algorithm are lightweight we think that our system could be able to run on real-time on a robotic platform.

The paper also compared two recent descriptors used for place recognition. GIST gives better results in the recognition of instances task (despite greater sensibility to global illumination) while CENTRIST gives better results for classification.

The average classification rate is a rough metric. The use of more sophisticated performance measures like the area under the ROC Curve (which is equivalent to the Wilcoxon test of ranks) could lead to indepth inspection of the results. 
Future works include a completely on-line algorithm i.e. replacing the SOM with an on-line, incremental algorithm. Several ideas could be used to overcome the explicit reset during recognition, which is a clear limitation of the current system. For instance, we could use a mechanism for detecting doorways. As we have generative, probabilistic classifiers, we could also use the uncertainty of the classification to allow automatic reset.

Our system is compatible with classical BoW techniques so, to improve the performance and especially the robustness to illumination, we can use an interest point based signature like SIFT.

To enforce the independence assumption we could use subsampling. Alternatively, using $n$-order Markov Model (phrases of visual prototypes) may be interesting because they could capture the timedependence of the visual prototypes during exploration. This would alleviate the independence assumption of our method.

\section{ACKNOWLEDGEMENT}

We thanks David Filliat for fruitful discussion and code exchange, Mohamed Jaite for setting-up the COLD database and Andrzej Pronobis for helping us with the COLD database.

\section{REFERENCES}

Csurka, G., Bray, C., Dance, C., and Fan, L. (2004). Visual categorization with bags of keypoints. In Workshop on Statistical Learning in Computer Vision, ECCV, pages $1-22$.

Douze, M., Jégou, H., Sandhawalia, H., Amsaleg, L., and Schmid, C. (2009). Evaluation of gist descriptors for web-scale image search. In International Conference on Image and Video Retrieval. ACM.

Fei-Fei, L. and Perona, P. (2005). A bayesian hierarchical model for learning natural scene categories. In IEEE Computer Society Conference on Computer Vision and Pattern Recognition, volume 2, pages 524531.

Filliat, D. (2008). Interactive learning of visual topological navigation. In Proceedings of the 2008 IEEE International Conference on Intelligent Robots and Systems (IROS 2008).

Filliat, D. and Meyer, J.-A. (2003). Map-based navigation in mobile robots - i. a review of localisation strategies. Journal of Cognitive Systems Research, 4(4):243-282.

Gokalp, D. and Aksoy, S. (2007). Scene classification using bag-of-regions representations. In IEEE Conference on Computer Vision and Pattern Recognition (CVPR 2007), pages 1-8, Minneapolis, USA.
Guillaume, H., Denquive, N., and Tarroux, P. (2005). Contextual priming for artificial visual perception. In European Symposium on Artificial Neural Networks (ESANN 2005), pages 545-550, Bruges, Belgium.

Kohonen, T. (1990). Improved versions of learning vector quantization. In International Joint Conference on Neural Networks, pages 545-550.

Lazebnik, S., Schmid, C., and Ponce, J. (2006). Beyond bags of features: Spatial pyramid matching for recognizing natural scene categories. In Association, I., editor, IEEE Conference on Computer Vision and Pattern Recognition, volume II, pages 2169-2178, New York.

Mozos, O. M. (2008). Semantic Place Labeling with Mobile Robots. PhD thesis, University of Freiburg, Freiburg, Germany.

Mozos, O. M., Stachniss, C., and Burgard, W. (2005). Supervised learning of places from range data using adaboost. In IEEE International Conference on Robotics and Automation, volume 2.

$\mathrm{Ng}$, A. Y. and Jordan, M. I. (2002). On discriminative vs. generative classifiers: A comparison of logistic regression and naive bayes. In Advances in Neural Information Processing Systems, volume 14. MIT Press.

Ni, K., Kannan, A., Criminisi, A., and Winn, J. (2009). Epitomic location recognition. IEEE Transactions on Pattern Analysis and Machine Intelligence, 31(12):2158-2167.

Ojala, T., Pietikäinen, M., and Mäenpää, T. (2002). Multiresolution gray-scale and rotation invariant texture classification with local binary patterns. IEEE Transactions on Pattern Analysis and Machine Intelligence, pages $971-987$.

Oliva, A. and Torralba, A. (2001). Modeling the shape of the scene: A holistic representation of the spatial envelope. International Journal of Computer Vision, 42(3):145-175.

Orabona, F., Castellini, C., Caputo, B., Luo, J., and Sandini, G. (2007). Indoor place recognition using online independent support vector machines. In Proceeding of the British Machine Vision Conference (BMVC 2007), pages 1090-1099, Warwick, UK.

Pronobis, A. and Caputo, B. (2007). Confidence-based cue integration for visual place recognition. In Proceedings of the IEEE/RSJ International Conference on Intelligent Robots and Systems (IROS 2007), San Diego, CA, USA.

Pronobis, A. and Caputo, B. (2009). Cold: Cosy localization database. The International Journal of Robotics Research, 28(5).

Pronobis, A., Caputo, B., Jensfelt, P., and Christensen, H. I. (2006). A discriminative approach to robust visual place recognition. In Proceedings of the IEEE/RSJ International Conference on Intelligent Robots and Systems (IROS 2006), pages 3829-3836, Beijing, China.

Pronobis, A., Mozos, O. M., Caputo, B., and Jenseflt, P. (2010). Multi-modal semantic place classification. The International Journal of Robotics Research, 29(2$3): 298-320$. 
Quattoni, A. and Torralba, A. (2009). Recognizing indoor scenes. In IEEE Conference on Computer Vision and Pattern Recognition.

Torralba, A. (2003). Contextual priming for object detection. International Journal of Computer Vision, 53(2):169-191.

Torralba, A., Murphy, K., Freeman, W., and Rubin, M. (2003). Context-based vision system for place and object recognition. Technical report, Cambridge, MA.

Ullah, M. M., Pronobis, A., Caputo, B., Luo, J., Jensfelt, P., and Christensen, H. I. (2008). Towards robust place recognition for robot localization. In Proceedings of the IEEE International Conference on Robotics and Automation (ICRA 2008), Pasadena, USA.

Vasudevan, S. and Siegwart, R. (2008). Bayesian space conceptualization and place classification for semantic maps in mobile robotics. Robotics and Autonomous Systems, 56(6):522-537.

Walker, L. and Malik, J. (2004). When is scene identification just texture recognition? Vision Research, 44:2301-2311.

Wu, J., Christensen, H., and Rehg, J. (2009). Visual place categorization: Problem, dataset, and algorithm. In IEEE/RSJ International Conference on Intelligent Robots and Systems, 2009 (IROS 2009), pages 47634770, St. Louis, USA. IEEE. 